\title{
Peptide Release on the Ribosome Involves Substrate-Assisted Base Catalysis
}

\author{
Masoud Kazemi, ${ }^{\dagger}$ Fahmi Himo, ${ }^{*}, *$ and Johan Åqvist ${ }^{*}{ }^{\dagger}$ \\ ${ }^{\dagger}$ Department of Cell and Molecular Biology, Uppsala University, Box 596, BMC, SE-751 24 Uppsala, Sweden \\ ${ }^{\ddagger}$ Department of Organic Chemistry, Arrhenius Laboratory, Stockholm University, SE-106 91 Stockholm, Sweden
}

\section{Supporting Information}

ABSTRACT: Termination of protein synthesis on the ribosome involves hydrolysis of the ester bond between the P-site tRNA and the nascent peptide chain. This reaction occurs in the peptidyl transferase center and is triggered by the class I release factors RF1 and RF2 in prokaryotes. Peptidyl-tRNA hydrolysis is $\mathrm{pH}$-dependent, and experimental results suggest that an ionizable group with $\mathrm{p} K_{\mathrm{a}}>9$ is involved in the reaction. The nature of this group is, however, unknown. To resolve this problem, we conducted density functional theory calculations using a large cluster model of the peptidyl transferase center. Our calculations reveal that peptidyl-tRNA hydrolysis occurs via a base-catalyzed mechanism with a predicted activation energy of $15.8 \mathrm{kcal} \mathrm{mol}^{-1}$, which is in good agreement with experimental data. In this mechanism, the P-site A76 2'-OH group is deprotonated and acts as the general base by activating the nucleophilic water molecule. The energy cost of deprotonating the $2^{\prime}$-hydroxyl group at $\mathrm{pH} 7.5$ is estimated to be about $8 \mathrm{kcal} \mathrm{mol}^{-1}$, on the basis of its experimental $\mathrm{p} K_{\mathrm{a}}$ in aqueous solution, and this step is predicted to be the source of the observed $\mathrm{pH}$ dependence.

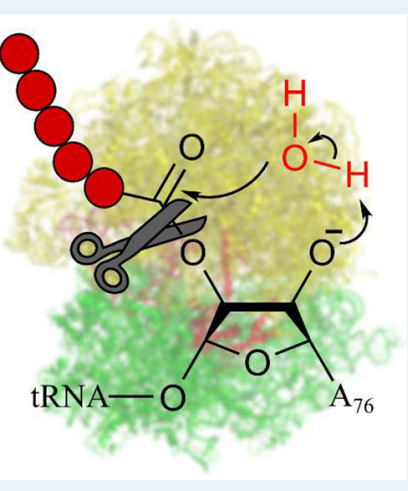
The proposed mechanism is consistent not only with experimentally derived activation energies but also with the observed kinetic solvent isotope effect.

KEYWORDS: ribosome, translation termination, release factor, peptidyl-tRNA hydrolysis, density functional theory

\section{INTRODUCTION}

Termination of mRNA translation on the ribosome occurs when a stop codon is presented in the A-site of the ribosomal decoding center. In bacteria, the three stop codons are recognized by two class I release factors (RFs), where RF1 reads UAA and UAG, while RF2 reads the UAA and UGA codons. Binding of the RFs to the decoding center triggers hydrolysis of the ester bond between the newly synthesized polypeptide and the last tRNA, residing in the P-site of the ribosome. This reaction takes place in the peptidyl transferase center (PTC), located some $75 \AA$ away from the decoding site, and is stimulated by the universally conserved GGQ motif in domain 3 of the RFs. The GGQ loop inserts the glutamine side chain into the PTC, where it positions a water molecule for nucleophilic attack on the ester carbonyl carbon, and the glutamine backbone also stabilizes the developing negative charge on the ester carbonyl oxygen. ${ }^{1-5}$ The PTC catalyzes two distinct reactions (Figure 1): namely, peptide bond formation (ester aminolysis) during the elongation phase of protein synthesis and peptidyl-tRNA hydrolysis (ester hydrolysis) in the termination phase. Both of these reactions thus involve cleavage of the P-site peptidyl-tRNA ester bond to the $3^{\prime}$ oxygen of A76, but with different nucleophiles. Nucleophilic attack of the $\alpha$-amino group of the A-site aminoacyl-tRNA on the ester carbonyl results in peptidyl transfer, while attack of water at the same position results in translation termination. Density functional theory (DFT) calculations predicted that the rate-limiting step for peptide bond formation is breaking of

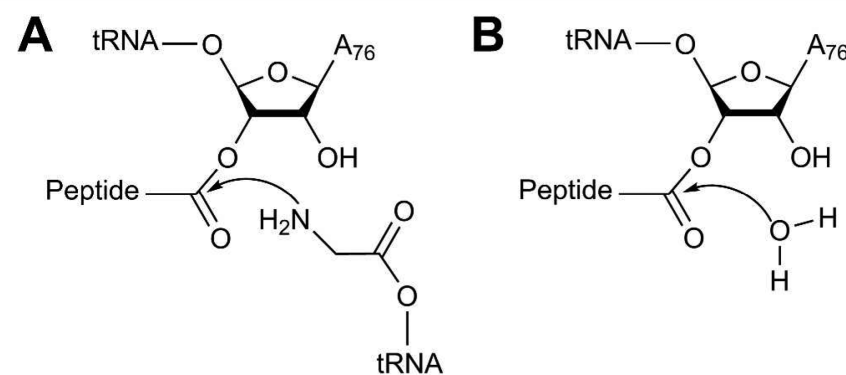

Figure 1. Different reactions catalyzed by the ribosomal peptidyl transferase center. The PTC catalyzes peptide transfer (A) during the elongation phase and peptidyl-tRNA ester hydrolysis (B) in termination of translation.

the $\mathrm{C}-\mathrm{O}$ bond in an eight-membered transition state (TS) involving a double proton shuttle. ${ }^{6}$ This transition state is consistent with both the $\mathrm{pH}$-rate profile ${ }^{7}$ and the large observed kinetic solvent isotope effect (KSIE) of 8.2 for peptide bond formation. 8 ,9

By analogy, it would thus seem reasonable to assume that peptidyl-tRNA hydrolysis also proceeds by the same mechanism. However, recent experimental results seem to contradict such a view. First, the $\mathrm{pH}$ dependence of the hydrolysis reaction

Received: October 5, 2016

Revised: November 3, 2016

Published: November 10, 2016 
A

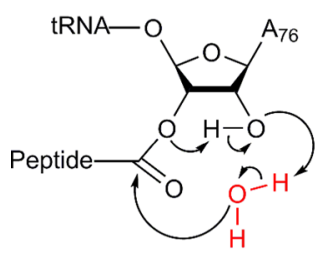

C

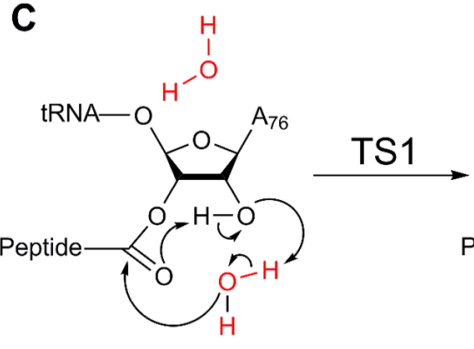

$\mathrm{R}$
B

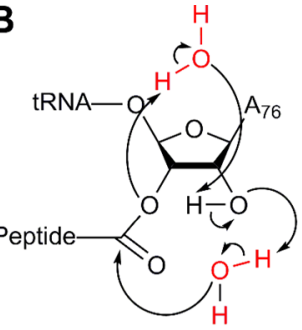

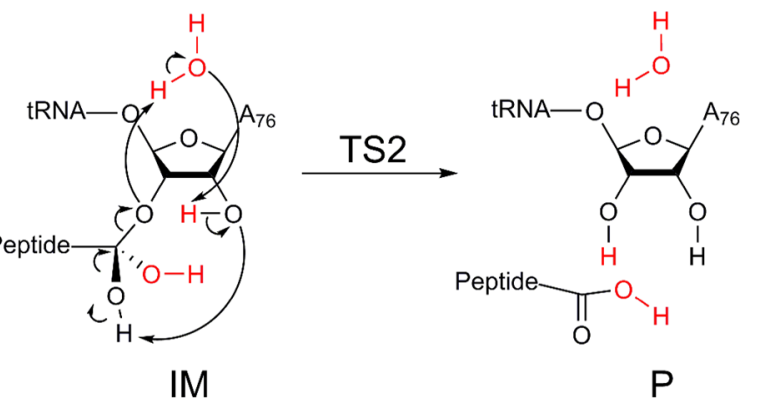

Figure 2. Schematic representation of previously proposed mechanisms for peptidyl-tRNA ester hydrolysis. In the concerted six- (A) and eightmembered (B) transition states the proton transfer occurs simultaneously with nucleophilic attack of water and ester bond fission. The neutral stepwise mechanism (C) proceeds via a neutral tetrahedral intermediate, which is formed by nucleophilic attack of a water molecule on the carbonyl carbon and concerted proton transfer to carbonyl oxygen. Collapse of the intermediate yields the product, and in this step one proton is transferred from the carbonyl oxygen to A76 $\mathrm{O}^{\prime}$ in a concerted eight-membered TS.

is distinctly different from that of peptide bond formation, which may be interpreted such that hydroxide ion rather than neutral water is the nucleophile. ${ }^{9-11}$ Such a mechanism was, in fact, modeled in earlier computer simulations of the termination reaction with a dA76 peptidyl-tRNA substrate, where very similar relative rates were found for various RF1 mutants in comparison to the neutral water mechanism. ${ }^{10}$ If the observed $\mathrm{pH}$ dependence does not reflect ionization of either the water nucleophile or the $2^{\prime}-\mathrm{OH}$ group of $\mathrm{A} 76$, the reaction would have to be dependent on deprotonation of some other ribosomal group, but there is no obvious candidate. Moreover, the $\mathrm{p} K_{\mathrm{a}}$ in water of the $2^{\prime}-\mathrm{OH}$ group in $3^{\prime}$-O-methyladenosine ${ }^{12}$ has been shown to be as low as 13.7 , which could make this group a viable candidate for ionization and base catalysis. Moreover, proton inventory experiments and the measured KSIE value of 4.1 for the hydrolysis reaction appear to be inconsistent with an eight-membered TS. ${ }^{9}$ Hence, the above experimental observations clearly suggest a change of mechanism for peptidyl-tRNA hydrolysis in comparison to peptide bond formation.

In recent theoretical studies of peptidyl-tRNA hydrolysis, concerted and neutral stepwise mechanisms were studied by a small cluster model. ${ }^{13,14}$ The concerted six- and eightmembered transition states (Figure 2A,B) are similar to those in peptide bond formation, ${ }^{6}$ except that the nucleophile in this case is a water molecule. As mentioned above, the $\mathrm{pH}$-rate profile and KSIE are distinctly different in peptidyl-tRNA hydrolysis and peptide bond formation. Therefore, a concerted TS does not seem to explain the underlying mechanism of the hydrolysis reaction. The proposed neutral stepwise mechanism (Figure 2C) is also similar to previously suggested transition states for peptide bond formation in which the carbonyl oxygen of the P-site substrate is protonated by the P-site $2^{\prime}-\mathrm{OH}$ group. $^{15,16}$ However, it should be noted that the highest resolution crystal structures of the PTC with nonreactive substrate analogues do not support such a proton transfer due to an unfavorable geometry. ${ }^{17-19}$ The key steps in the neutral mechanism $^{14}$ are the formation and collapse of a neutral tetrahedral intermediate, which both occur through a concerted proton transfer (Figure 2C). Hence, similar to the concerted transition states, the neutral stepwise mechanism is also not consistent with the $\mathrm{pH}$-rate profile of peptidyl-tRNA hydrolysis. Furthermore, the calculated activation energies for all studied mechanisms were too high in comparison to the experimental value ${ }^{9,11}$ and the model used in these studies did not include any parts of the rRNA in the PTC. ${ }^{13,14}$

The main goal of this work is to put forward a mechanism for peptidyl-tRNA hydrolysis that is compatible with the experimental observations: namely the $\mathrm{pH}$ dependence, KSIE, and activation energy. To achieve this goal, we constructed a large cluster model ( 220 atoms) of the PTC which was used to study possible mechanisms by the density functional theory approach. To be able to compare our proposed mechanism to earlier suggestions, we also reexamined the previously proposed mechanisms by our cluster model. For each mechanism the KSIE was calculated for the rate-limiting step, which together with the calculated activation energies can be compared directly to the available experimental data. The results of our calculations suggest that the $\mathrm{P}$-site $\mathrm{A} 76 \mathrm{O} 2$ ' releases a proton to the bulk and acts as a general base in activating the nucleophilic water. The nucleophilic attack of water with simultaneous proton transfer to the deprotonated $\mathrm{A} 76 \mathrm{O}^{\prime}$ is the only mechanism that is compatible with the measured activation energy and the magnitude of the KSIE. Moreover, the source of the $\mathrm{pH}$ dependence of translation termination seems to be the deprotonation of the $2^{\prime}-\mathrm{OH}$ group of the P-site tRNA rather than water.

\section{COMPUTATIONAL METHODS}

The peptidyl transferase center was represented by a cluster model based on the most recent crystal structure of RF2 bound to $70 \mathrm{~S}$ ribosome $\mathrm{e}^{20}$ (PDB code $\left.4 \mathrm{~V} 5 \mathrm{~J}\right)$. The substrate analogue 


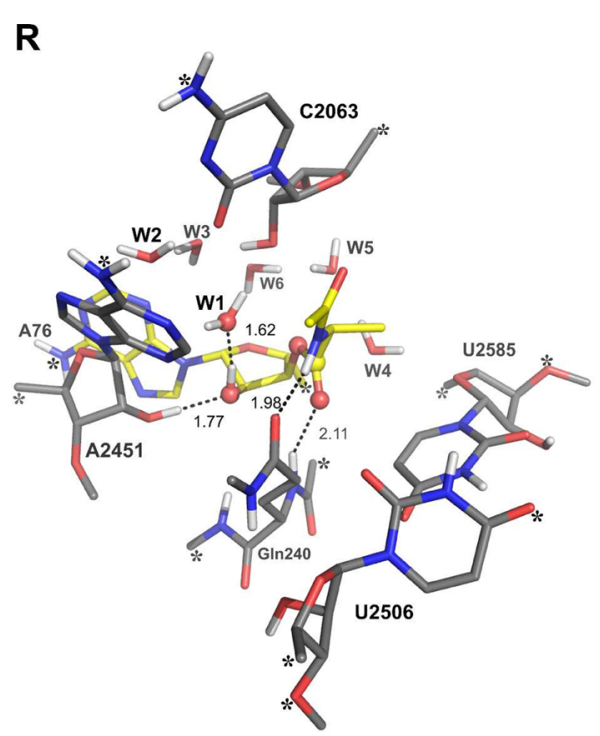

$\mathbf{R}^{\prime}$

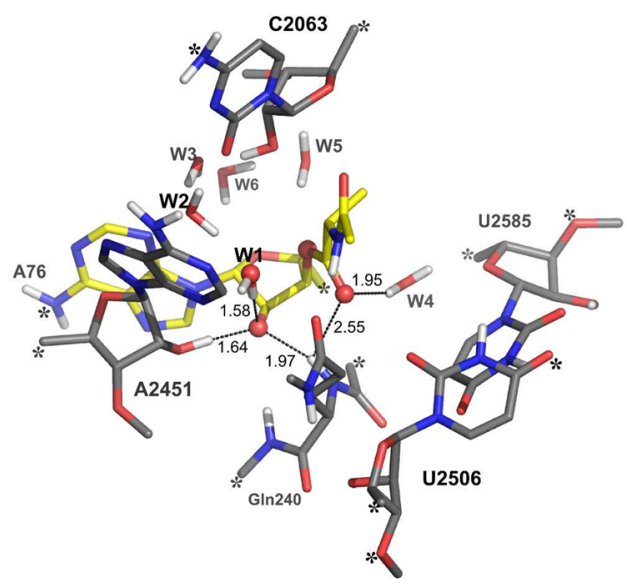

Figure 3. Computational model of the peptidyl transferase center, showing the optimized structure of the reactant state (R) and the deprotonated reactant state $\left(R^{\prime}\right)$. $R$ is used as the reference state for the six- and eight-membered and neutral stepwise mechanisms, while $R^{\prime}$ is the reference state for the base-catalyzed mechanism. The nucleophilic water is labeled as W1 and makes a hydrogen bond to the A76 2'-OH group. Fixed atoms are marked with asterisks.

(Phe-NH-A76) was transformed to a natural substrate (Ala-OA76), and the terminal amine group of the substrate was acylated to mimic the next peptide bond. The nucleotides C2063, A2451, U2506, and U2585 from the 50S subunit and the conserved methylated Gln240 residue from RF2 were included in the model. The $\mathrm{O5}^{\prime}$ atoms of the nucleotides were capped with a methyl group to avoid artificial hydrogen bonds. Six water molecules were included in the model on the basis of similar crystal structures of the PTC ${ }^{18,19}$ and previous empirical valence bond (EVB) calculations. ${ }^{1,5}$ To account for the steric effect of surrounding parts of the ribosome, some atoms were kept fixed at their crystallographic position during geometry optimizations (Figure 3). The final model after addition of hydrogen atoms is composed of 224 atoms.

DFT calculations were performed with the Gaussian 09 package. $^{21}$ Stationary points were optimized with the M06-2X functional $^{22}$ and $6-31 \mathrm{G}(\mathrm{d}, \mathrm{p})$ basis set. The electronic energies were then obtained by single-point calculations using M06-2X and the $6-311+\mathrm{G}(2 \mathrm{~d}, 2 \mathrm{p})$ basis set. Zero-point energies (ZPE) were obtained by frequency calculations on the optimized structures using the same level of theory. To account for the dielectric effect of the ribosome environment, solvation effects were calculated by the SMD continuum solvation model ${ }^{23}$ with three different dielectric constants $(4,24$, and 78$)$ at the same level of theory as in the geometry optimization. The final energies reported are the electronic energies with the large basis set which are corrected for ZPE and solvation effects.

\section{RESULTS AND DISCUSSION}

Validity of the PTC Model. The peptidyl transferase center was represented by a cluster model based on the crystal structure of RF2 bound to the $70 \mathrm{~S}$ ribosome ${ }^{20}$ (PDB code 4V5J). The rRNA bases C2063 and A2451 were included in the model, since they participate in hydrogen bonds with crystallographically observed water molecules and the P-site A76 2'-OH group (Figure 3). Although the rRNA residues $\mathrm{U} 2506$ and U2585 do not interact directly with the reactive fragments, they make up the first shell of the PTC and possibly dictate the conformation of the nascent peptide chain.
Mutation experiments support the role of these residues in translation termination, ${ }^{24}$ and they were therefore also included in the model. The conserved methylated Gln240 RF side chain plays a crucial role in peptidyl-tRNA hydrolysis, ${ }^{25-27}$ and it positions the nucleophilic water in close proximity to the substrate. ${ }^{1,5}$ The main-chain amide group of this residue also acts by stabilizing the developing negative charge on the substrate carbonyl oxygen at the transition state. ${ }^{1-5}$ For this reason, both the methylated side chain and the main chain were included in the cluster model.

The resolution of the crystal structure $(3.1 \AA)^{20}$ is inadequate to provide reliable information about the positions of water molecules in the PTC. To acquire an accurate description of the PTC, six water molecules (W1-W6 in Figure 3) were therefore added to the model by comparison of the RF2ribosome complex (PDB code 4V5J) with a higher resolution structure of the $50 \mathrm{~S}$ subunit with substrate analogues ${ }^{18,19}$ (PDB codes 1VQN, 1VQP) and with previous EVB simulations of peptidyl-tRNA hydrolysis. ${ }^{1,5}$ Waters $\mathrm{W} 1-\mathrm{W} 3$ were thus adopted from the crystal structures, while W4 and W5 were taken from the earlier molecular dynamics (MD) simulations and W6 was added to shield the active site from the continuum model. During the geometry optimizations it turns out that the last water molecule, in fact, occupies the same position as A75 $\mathrm{O} 2^{\prime}$ in the P-site. Overall, this model consists of 224 atoms with a net charge of 0 .

At the periphery of the system some atoms were kept fixed in order to maintain the geometry imposed by the surrounding part of the structure (Figure 3). During initial geometry optimizations, the substrate (acylated alanine ester) rotated from the initial position by $\sim 60^{\circ}$ around the ester bond in comparison to the X-ray crystal structure ${ }^{20}$ (Figure S1 in the Supporting Information). The orientation of the substrate analogue in this crystal structure is, however, most likely a suboptimal model for hydrolysis reaction. That is, not only is the electron density weak for the peptide part (Figure S2 in the Supporting Information) but also the substrate analogue has a (possibly positively charged) terminal amine group which would normally be far away from the PTC with a longer 

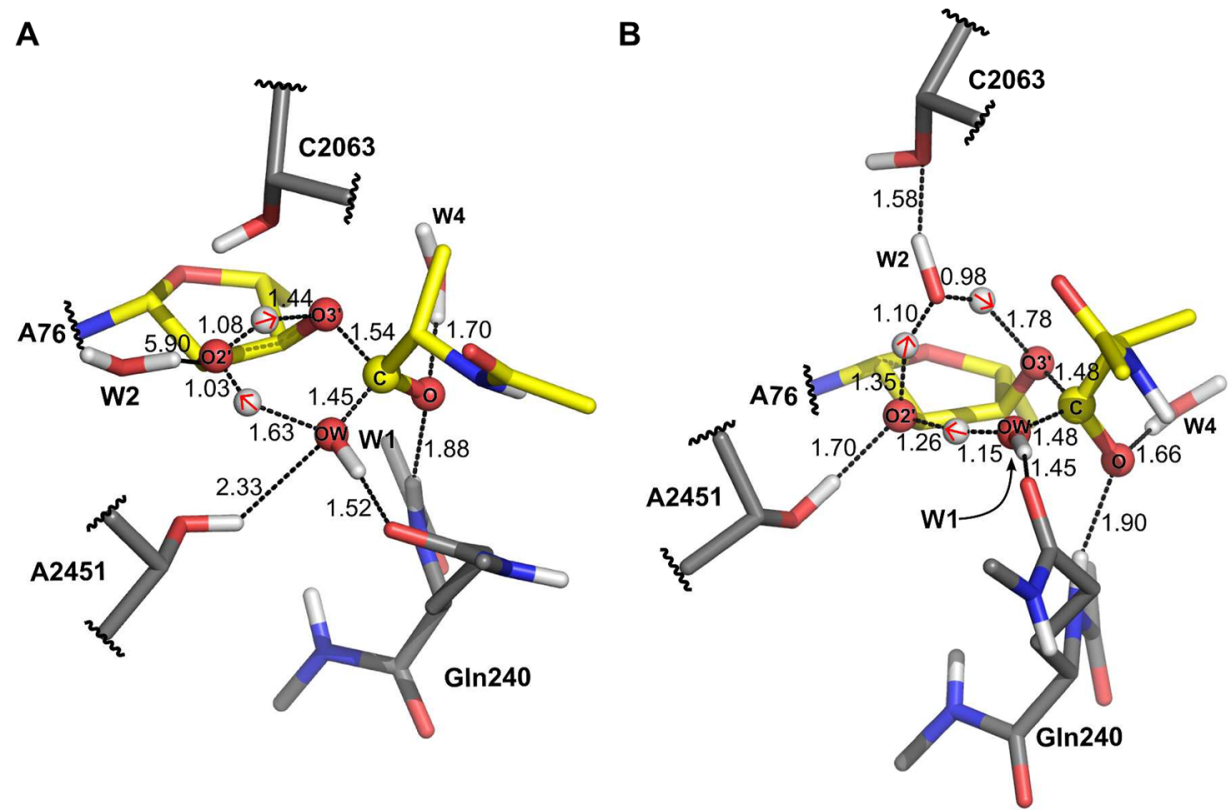

Figure 4. Optimized structures of the concerted six-membered (A) and eight-membered (B) transition states. The oxygen of nucleophilic water is labeled as OW. In both transition states the side chain of Gln240 interacts with the nucleophilic water. The developing charge on the substrate carbonyl oxygen is stabilized by the backbone amide of Gln240 and W4 (distances in $\AA$ ). Only residues in the first solvation shell are shown, and nonpolar hydrogen atoms are omitted for clarity.

\section{Table 1. Calculated Energetics for Different Peptidyl-tRNA Hydrolysis Mechanisms ${ }^{a}$}

\begin{tabular}{|c|c|c|c|c|c|c|c|c|}
\hline & $\mathrm{R}^{\prime}$ & TS1 & $\mathrm{IM}$ & TS2 & $\mathrm{P}^{\prime}$ & $\mathrm{P}$ & $\Delta \mathrm{ZPE}$ & KSIE \\
\hline base catalyzed & 8.4 & 15.8 & 6.9 & 9.5 & 5.3 & -9.6 & 0.42 & 3.4 \\
\hline neutral stepwise & & 23.4 & 5.1 & 30.4 & & & 0.71 & 2.7 \\
\hline eight-membered concerted & & 33.0 & & & & & 1.30 & 6.8 \\
\hline six-membered concerted & & 41.1 & & & & & 0.59 & 2.4 \\
\hline
\end{tabular}

${ }^{a}$ All energies are in $\mathrm{kcal} \mathrm{mol}^{-1}$ and are relative to state $\mathrm{R}$. The solvation contribution of the reported energies is calculated by a dielectric constant of 24 in the SMD model. The experimentally measured activation enthalpy ${ }^{9}$ and $\mathrm{KSIE}^{9}$ are $15.9 \mathrm{kcal} \mathrm{mol}^{-1}$ and 4.1 , respectively. For the base-catalyzed mechanism, the energetic cost of initial deprotonation was estimated from the $\mathrm{p} K_{\mathrm{a}}$ of $\mathrm{A} 762^{\prime}-\mathrm{OH}$ (see text).

substrate. In addition, the ester oxygen is replaced by an $\mathrm{NH}$ group, as in several other crystallized analogues, which changes its polarity. ${ }^{28}$ On the other hand, the orientation in our model very closely matches the most relevant crystal structure with bound P-site substrate in the peptidyl transfer reaction ${ }^{18}$ (PDB code $1 \mathrm{VQN}$ ), which also contains a longer peptide chain (Figure S1B). In this conformation, the carbonyl oxygen of the substrate forms a hydrogen bond with the backbone amide of the conserved Gln240 residue (Figure 3). Since translation termination involves a full-length peptide chain, this orientation of the substrate appears to be a more reasonable representation for peptidyl-tRNA hydrolysis in comparison to that observed in the crystal structure of RF2 bound to the $70 \mathrm{~S}$ ribosome $\mathrm{e}^{20}$ (Figure S1A).

Different Possible Hydrolysis Mechanisms. Any proposed mechanism for peptidyl-tRNA hydrolysis on the ribosome must be compatible both with the experimental activation free energy of $18 \mathrm{kcal} \mathrm{mol}^{-1}$ and the observed $\mathrm{pH}$ dependence of this reaction. ${ }^{9,11,26}$ In the concerted six- and eight-membered transition states, however, the nucleophilic attack of the water molecule occurs simultaneously with proton transfer to the P-site $\mathrm{O}^{\prime}$ and ester bond fission (Figure 2A,B). Hence these TSs do not explain the $\mathrm{pH}$ dependence of peptidyl-tRNA hydrolysis. Nevertheless, we optimized these transition states (Figure 4) to compare various possible mechanisms within the same computational model. The activation energies for the six- and eight-membered TSs are 41.1 and $33.0 \mathrm{kcal} \mathrm{mol}^{-1}$, respectively (Table 1). The lower activation energy of the eight-membered TS is due to participation of the extra water (W2 in Figure 4B) in the proton relay, which relieves enthalpic strain in the transition state. More favorable dipole interactions may also contribute. ${ }^{29}$ The same effect was also observed in previous studies of peptide bond formation and peptidyl-tRNA hydrolysis. ${ }^{6,13}$ In the eight-membered TS, the O2' atoms of C2063 and A2451 form hydrogen bonds with $\mathrm{W} 2$ and $\mathrm{A} 76 \mathrm{O}^{\prime}$, respectively (Figure 4B). These interactions are, however, lost in the sixmembered TS (Figure 4A) and A2451 O2' instead interacts with the attacking water (W1). In both transition states the developing charge on the carbonyl oxygen of the substrate (O in Figure 4) is stabilized by hydrogen bonding to W4 and the backbone amide of the conserved Gln240. The side chain of Gln240 has similar conformations in two different TSs, which are consistent with earlier crystal structures ${ }^{2-4,20}$ and the predictions from MD simulations. ${ }^{1,5}$ Most importantly, the carbonyl oxygen of the Gln 240 side chain hydrogen bonds to the nucleophilic water molecule (Figure 4), thereby orienting it for attack on the substrate as predicted earlier. ${ }^{1,5}$

The proposed neutral stepwise mechanism ${ }^{14}$ proceeds through formation of a neutral tetrahedral intermediate (Figure 2C). The intermediate (IM) is formed by nucleophilic attack of the catalytic water molecule on the substrate carbonyl carbon 

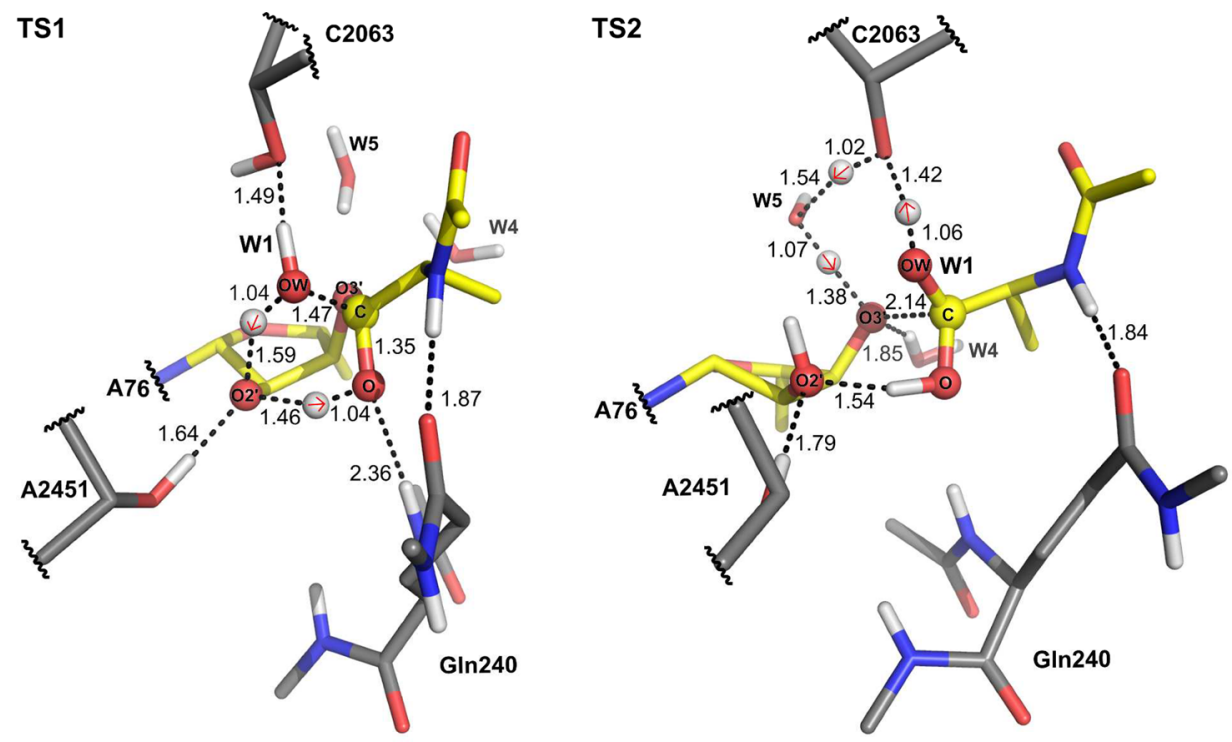

Figure 5. Transition states of the neutral stepwise mechanism. Formation of the tetrahedral intermediate occurs via a six-membered transition state (TS1) in which the carbonyl oxygen (O) is protonated. The ester bond breaks with simultaneous proton transfer from the attacking water to O3' in an eight-membered transition state (TS2). Only residues in the first solvation shell are shown, and nonpolar hydrogen atoms are omitted for clarity.

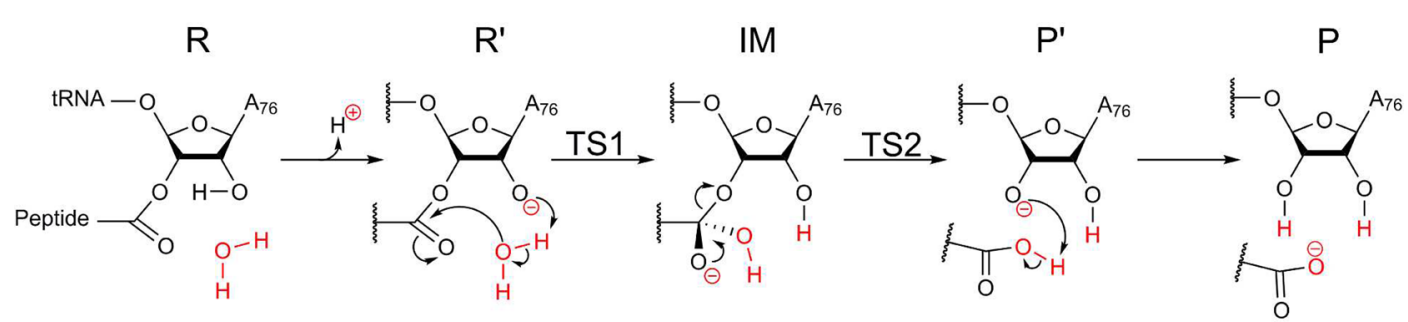

Figure 6. Schematic representation of the base-catalyzed mechanism. Following the initial deprotonation of the A76 2'-OH group, the tetrahedral intermediate (IM) is formed by nucleophilic attack of a water molecule with simultaneous proton transfer to A76 O2' (TS1). Ester bond fission occurs in the second transition state (TS2), which yields the protonated product.
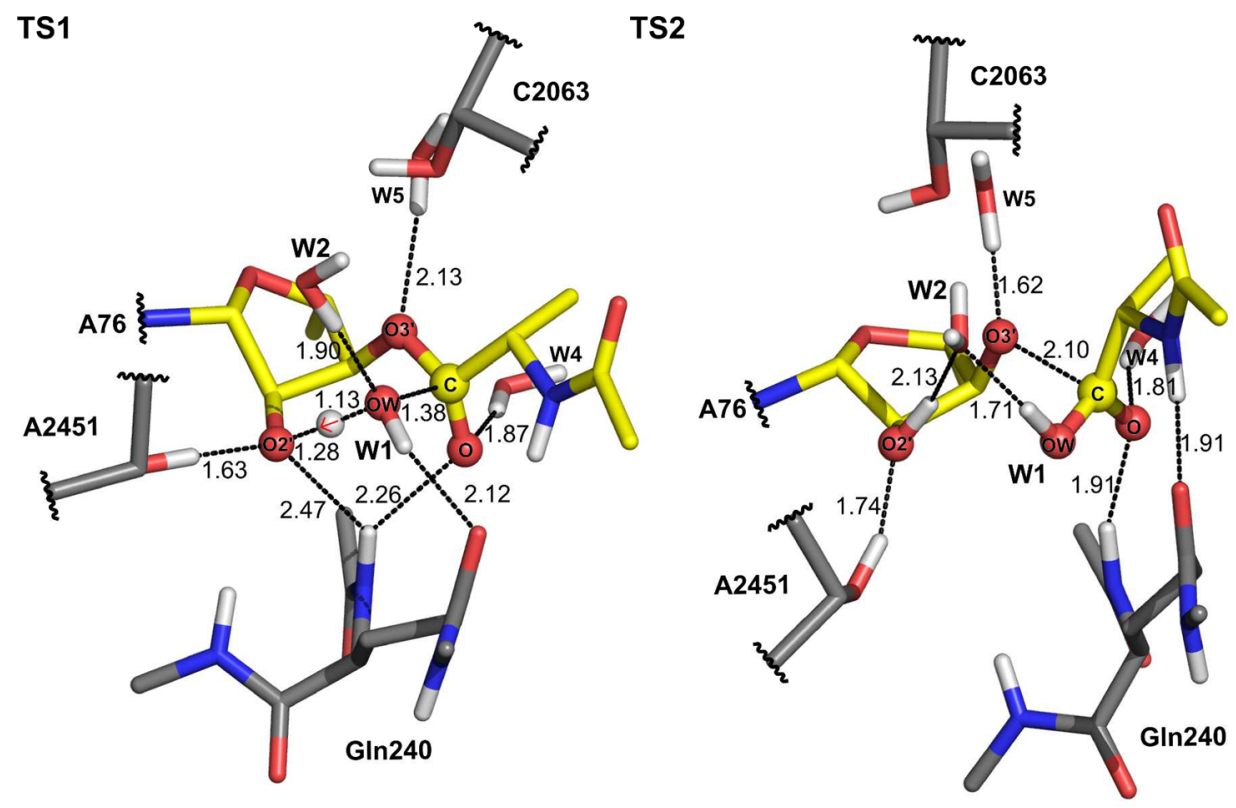

Figure 7. Transition states for the base-catalyzed mechanism. The optimized structures of the TSs for tetrahedral intermediate formation (TS1) and decomposition (TS2) are shown. The side chain of Gln240 interacts with the attacking water in TS1 and with the leaving peptide amide in TS2. In both transition states, the Gln240 backbone amide and W4 stabilize the negative charge of carbonyl oxygen (O). Only residues in the first solvation shell are shown, and nonpolar hydrogen atoms are omitted for clarity. 
with simultaneous proton transfer from the attacking water to the carbonyl oxygen (TS1 in Figure 5). This proton transfer is mediated by the P-site $\mathrm{A} 762^{\prime}-\mathrm{OH}$ group, which results in a sixmembered TS with an activation energy of $23.4 \mathrm{kcal} \mathrm{mol}^{-1}$. However, the proton transfer from $\mathrm{O}_{2}$ ' to the substrate carbonyl oxygen requires a significant rotation of the substrate, which is not seen in any crystal structures, and there is no interaction between the Glu240 side chain and the reacting groups. In the second step, the $\mathrm{C}-\mathrm{O}$ ester bond breaks and one proton is transferred simultaneously from IM to the P-site A76 O3' (Figure 2C), where it was suggested that the proton transfer would again be mediated by $\mathrm{A} 76 \mathrm{O}^{\prime}$ and a water molecule. However, the predicted activation energy barrier ${ }^{14}$ for this step was over $37 \mathrm{kcal} \mathrm{mol}^{-1}$. In our PTC model, we observed that the $2^{\prime}-\mathrm{OH}$ group of $\mathrm{C} 2063$ is better positioned to relay the proton from IM to the P-site A76 O3'. Since the $\mathrm{C} 20632^{\prime}-\mathrm{OH}$ is chemically equivalent to the P-site A76 2'-OH, we optimized a transition state in which the proton transfer is mediated by the former group and an auxiliary water (TS2 in Figure 5). The activation energy for this transition state is 30.4 $\mathrm{kcal} \mathrm{mol}^{-1}$, which is approximately $8 \mathrm{kcal} \mathrm{mol}^{-1}$ lower than the alternative neutral mechanism. ${ }^{14}$ Overall, the activation energy of this mechanism is very similar to that obtained from the concerted eight-membered TS (Table 1), indicating that initial protonation of substrate carbonyl oxygen does not yield any notable catalytic effect. It can further be noted that there is no significant entropy contribution observed for this reaction experimentally that could bring the free energy barrier down to the observed value.'

Base-Catalyzed Mechanism. The $\mathrm{pH}$ dependence of peptidyl-tRNA hydrolysis indicates that an ionizable group with $\mathrm{pK}_{\mathrm{a}}>9$ participates in the reaction. ${ }^{9,11}$ This suggested that hydroxide ion could in fact act as the nucleophile in the hydrolysis reaction. ${ }^{9-11}$ To investigate this, we replaced the attacking nucleophilic water with a hydroxide ion. In the geometry optimization, however, the hydroxide ion abstracts one proton from the A76 $2^{\prime}-\mathrm{OH}$ group. This proton transfer is consistent with a lower $\mathrm{p} K_{\mathrm{a}}$ of $\mathrm{A} 762^{\prime}-\mathrm{OH}$ in comparison to water, as was observed for the relevant model compound $3^{\prime}-O$ methyladenosine with $\mathrm{p} K_{\mathrm{a}}=13.70 .^{12}$ Hence the deprotonated $\mathrm{A} 76 \mathrm{O}^{\prime}$ state was taken as the reference state (state $\mathrm{R}^{\prime}$ in Figure 6) for the base-catalyzed hydrolysis. In this state, the negative charge on $\mathrm{A} 76 \mathrm{O}^{\prime}$ is stabilized by hydrogen bonding to the A2451 2'-OH group, $\mathrm{W} 1$, and the backbone amide of $\mathrm{Gln} 240$.

A negatively charged tetrahedral intermediate is then formed by the nucleophilic attack of $\mathrm{W} 1$ on the carbonyl group of the peptidyl-tRNA with simultaneous proton transfer to A76 O2' (Figure 6). The activation energy for this step (TS1) is found to be $7.4 \mathrm{kcal} \mathrm{mol}^{-1}$ relative to $\mathrm{R}^{\prime}$ (Figure 7). The negative charge of the tetrahedral intermediate is stabilized by hydrogen bonding to the backbone amide of $\mathrm{G} \ln 240$ and $\mathrm{W} 4$, as predicted earlier. ${ }^{1,5,10}$ This brings the relative energy of the intermediate down to $-1.5 \mathrm{kcal} \mathrm{mol}^{-1}$ in comparison to $\mathrm{R}^{\prime}$. Subsequently, fission of the bond between A76 O3' and the substrate carbon occurs almost spontaneously with only a minor $2.6 \mathrm{kcal} \mathrm{mol}^{-1}$ barrier (TS2 in Figure 7), thereby forming the protonated product (Figure 6). The activation and reaction energy for this step are thus $\Delta E^{\ddagger}=1.0 \mathrm{kcal} \mathrm{mol}^{-1}$ and $\Delta E^{\circ}=$ $-3.1 \mathrm{kcal} \mathrm{mol}^{-1}$ relative to the state $\mathrm{R}^{\prime}$, respectively. Proton transfer from the protonated product to the leaving A76 O3' (Figure 6) has a very low barrier and yields the final product, with a reaction energy of $-18.0 \mathrm{kcal} \mathrm{mol}^{-1}$ relative to $\mathrm{R}^{\prime}$.
However, to obtain the overall reaction energy of the basecatalyzed mechanism, the initial proton transfer from the A76 2 '-OH group to bulk solvent (at $\mathrm{pH} 7.5$ as in the experiments) must be taken into account. Such a calculation is, however, very difficult to carry out by any computational approach, since it is equivalent to evaluating the absolute energy of a (negative) charge in the complex ribosome environment, with numerous phosphate charges and counterions. ${ }^{10}$ Therefore, the most reasonable and unbiased estimate of the energy cost for this step is to assume that the $2^{\prime}-\mathrm{OH} \mathrm{p} K_{\mathrm{a}}$ value is unperturbed by the ribosome environment, as the crystal structures of the PTC give no reason to expect any unusually large $\mathrm{p} K_{\mathrm{a}}$ shifts. This is also supported by the small $\mathrm{p} K_{\mathrm{a}}$ shifts observed experimentally for the attacking amine in the peptidyl transfer reaction. ${ }^{7}$

Hence, the reaction free energy can then be estimated to be the same as in aqueous solution and is given by $\Delta G^{\circ}=$ $1.36\left(\mathrm{p} K_{\mathrm{a}}-\mathrm{pH}\right)$. This gives a free energy cost for the initial proton transfer step of $8.4 \mathrm{kcal} \mathrm{mol}^{-1}$ using the estimated $\mathrm{p} K_{\mathrm{a}}$ of 13.7 for the $\mathrm{A} 762^{\prime}-\mathrm{OH}$ group ${ }^{12}$ and a solution $\mathrm{pH}$ of 7.5 at $25{ }^{\circ} \mathrm{C}$. Although it could perhaps be argued that the PTC environment would not stabilize the $\mathrm{A} 76 \mathrm{O} 2^{\prime}$ anion as much as the solution phase, it should be noted that the PTC is highly preorganized by hydrogen bonding to the relevant groups, which reduces the entropy cost of solvent reorganization that accompanies the water reaction. ${ }^{30,31}$ Therefore, the free energy estimate of the initial deprotonation of $\mathrm{A} 76 \mathrm{O} 2^{\prime}$ appears as a very reasonable approximation. Hence, for the base-catalyzed mechanism the rate-limiting nucleophilic attack of water on the substrate carbonyl carbon (TS1) is predicted to have an overall activation energy of $15.8 \mathrm{kcal} \mathrm{mol}^{-1}$ relative to the state $\mathrm{R}$ (Figure 8). Considering that the experimentally determined

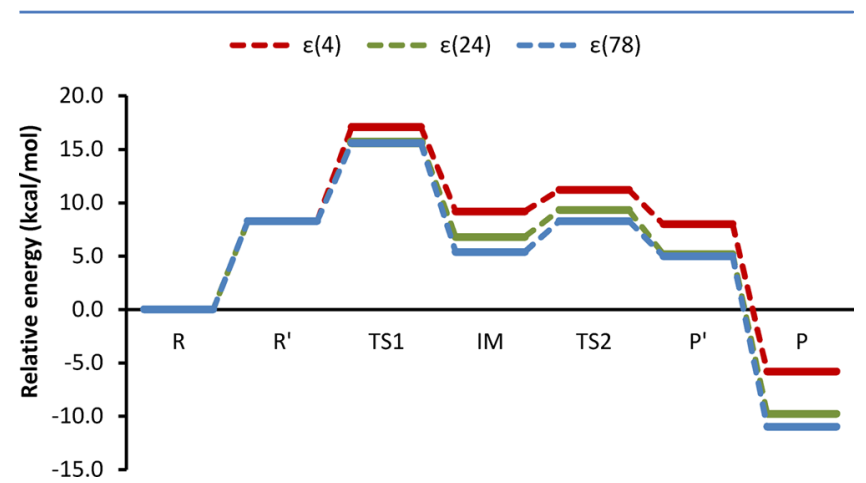

Figure 8. Energetics of the base-catalyzed peptide release mechanism. The calculated energy profile of the base-catalyzed mechanism with different dielectric constants of 4,24 , and 78 are shown.

entropy ${ }^{9}$ contribution is $-T \Delta S^{\ddagger}=1.6 \mathrm{kcal} \mathrm{mol}^{-1}$, this would yield a predicted free energy barrier of $17.4 \mathrm{kcal} \mathrm{mol}^{-1}$, which is in excellent agreement with the experimental data. ${ }^{9,11,26}$

In this mechanism, the deprotonated P-site A76 O2' thus acts as the general base that activates the nucleophilic water. The ionization of $\mathrm{A} 76 \mathrm{O}^{\prime}$ is therefore the most probable reason for the $\mathrm{pH}$ dependence of the hydrolysis reaction. This is also in agreement with mutation experiments of A76 O2' where elimination of the $2^{\prime}$-hydroxyl decreased the reaction rate by $\left(\sim 7 \times 10^{-5}\right)$-fold $\left(\sim 6 \mathrm{kcal} \mathrm{mol}^{-1}\right)$ in the RF1-catalyzed reaction. ${ }^{10}$ This decrease in the reaction rate would thus partially be due to the change of the general base from the Psite $\mathrm{A} 76 \mathrm{O}^{\prime}\left(\mathrm{p} K_{\mathrm{a}}=13.70\right)$ to water $\left(\mathrm{p} K_{\mathrm{a}}=15.74\right)$, which accounts for an $\sim\left(1 \times 10^{-2}\right)$-fold $\left(\sim 3 \mathrm{kcal} \mathrm{mol}^{-1}\right)$ decrease in the reaction rate. A similar effect was also, in fact, observed for 
the uncatalyzed ribosome reaction in the absence of release factor ${ }^{10}$ and on comparison of the hydrolysis of Leu-tRNA and Leu-ethyl ester in aqueous solution. ${ }^{32}$

Kinetic Solvent Isotope Effect. To further investigate the consistency of the proposed mechanisms with experimental observations, the KSIEs were calculated by replacing polar hydrogen atoms with deuterium (Table 1). In these calculations, only the ZPE contribution was taken into account, which has been shown to yield reliable results. ${ }^{33}$ For the concerted six- and eight-membered transition states, we obtained ZPE differences of 0.52 and $1.14 \mathrm{kcal} \mathrm{mol}^{-1}$ between the TSs and R which gives KSIEs of 2.4 and 6.8 for the six- and eight-membered TSs, respectively. In the neutral stepwise mechanism, the ZPE difference for TS2 is $0.58 \mathrm{kcal} \mathrm{mol}^{-1}$, which yields a KSIE of 2.7 for this transition state. For the ratelimiting step (TS1) of the base-catalyzed mechanism, the ZPE difference was found to be $0.46 \mathrm{kcal} \mathrm{mol}^{-1}$, which yields a KSIE of 2.16 relative to the state $R^{\prime}$. The equilibrium isotope effect (EIE) for the initial deprotonation step was estimated from the 2-chloroethanol dissociation equilibrium in water and deuterium oxide. That is, proton transfer from $\mathrm{ClCH}_{2} \mathrm{OH}(\mathrm{pK}$ $14.31)^{34}$ to bulk water at $\mathrm{pH} 7$ yields a reaction free energy of $10.0 \mathrm{kcal} \mathrm{mol}^{-1}$. For deuterated $\mathrm{ClCH}_{2} \mathrm{OD}\left(\mathrm{pK}_{\mathrm{a}}=14.31\right)^{33}$ at neutral $\mathrm{pD} 7.48$ the corresponding reaction free energy is 10.2 $\mathrm{kcal} \mathrm{mol}^{-1}$, which yields an EIE of 1.6. Using this value as an approximation for the EIE of initial substrate deprotonation on the ribosome, the overall isotope effect for the rate-limiting step in the base-catalyzed mechanism is found to be 3.4 (Table 1). Since the $\mathrm{p} K_{\mathrm{a}}$ of 2-chloroethanol is very similar to that of the Psite A76 $2^{\prime}-\mathrm{OH}$ group, $^{12}$ the calculated EIE for initial deprotonation is a reasonable approximation.

The calculated KSIE of 3.4 for the base-catalyzed mechanism is thus also in good agreement with the measured value for the RF2-catalyzed reaction ${ }^{9}$ of 4.1 , and it lies between the values for the six- and eight-membered concerted TSs. Furthermore, it is important to emphasize that the base-catalyzed mechanism is that yielding the lowest activation energy and this would be true even if the $\mathrm{A} 762^{\prime}-\mathrm{OH}$ group had a $\mathrm{p} K_{\mathrm{a}}$ as high as that of water, in which case the activation energy would increase by $3 \mathrm{kcal}$ $\mathrm{mol}^{-1}$. Importantly, it is also the only mechanism consistent with the observed $\mathrm{pH}$ dependence of the peptide release reaction. Hence, these results strongly support the basecatalyzed mechanism in the peptidyl-tRNA hydrolysis reaction.

\section{CONCLUSION}

The peptidyl-tRNA hydrolysis reaction is one of the two processes catalyzed by the ribosomal peptidyl transferase center, and it is required for release of the newly synthesized polypeptide in termination of protein synthesis. Despite the importance of this reaction and a wealth of experimental results, the exact chemical mechanism has remained puzzling. In contrast to peptide bond formation, the peptide release rate is dependent on hydroxide ion concentration in the RF1- and RF2-catalyzed reactions. ${ }^{9,11}$ This suggests that ionization of either the nucleophilic water or P-site A76 2'-OH group is required for efficient peptidyl-tRNA hydrolysis. Among the various possible reaction schemes, our calculations now show that the mechanism in which ribosome "waits" for spontaneous ionization of the P-site A76 2'-OH group is that which gives the closest agreement with the experimentally derived activation free energy. Importantly, it is also the only proposed mechanism that can account for the observed $\mathrm{pH}$ dependence of the reaction. We further note that this conclusion does not really depend on our assumption of an unperturbed $2^{\prime}-\mathrm{OH} \mathrm{p} K_{\mathrm{a}}$ since the base-catalyzed mechanism is predicted to be favored by as much as $11 \mathrm{p} K_{\mathrm{a}}$ units.

Interestingly, $\mathrm{MD} / \mathrm{EVB}$ simulations of this type of mechanism could also explain the effects of RF1 mutations on the termination reaction with a P-site dA76 substrate, which shows a similar $\mathrm{pH}$ dependence. ${ }^{10}$ Here, it may also be noted that earlier $\mathrm{MD} / \mathrm{EVB}$ simulations of the termination reaction gave a large catalytic effect on the ribosome also for a neutral mechanism. ${ }^{1,5,10}$ This is perhaps not so strange, considering that the EVB transition state structures for neutral and anionic mechanisms were very similar, apart from the removal of one proton from the system. ${ }^{10}$ That is, the stabilizing effect from the Gln240 side chain and backbone, as well as the key water molecule, W4, is conserved for mechanisms that do not involve protonation of the substrate carbonyl (Figures 4 and 7). The absolute EVB free energy barrier is, however, dependent on the calibration against the uncatalyzed solution reaction, for which there are no unambiguous data regarding the difference in activation barriers between hydroxide and neutral water attack mechanisms. On the basis of a study of the spontaneous hydrolysis of Leu-tRNA and Leu-ethyl esters in aqueous solution, it was suggested that the mechanism is indeed dominated by hydroxide ion attack. ${ }^{32}$ However, in this case the leucine amine is protonated, which could facilitate nucleophilic attack, and the postulated mechanism is kinetically indistinguishable from water attack on the neutral ester. Nevertheless, in view of the recently reported linear $\mathrm{pH}$ dependence ${ }^{9}$ also of the uncatalyzed hydrolysis of fMet-tRNA ${ }^{\text {fMet }}$ (with no charged amine) it is likely that the spontaneous hydrolysis of a peptidyltRNA ester would also follow the substrate-assisted mechanism suggested herein.

Hence, in this mechanism the deprotonated A76 2'-OH group acts as a general base that activates the attacking water. In the rate-limiting step (TS1) the nucleophilic attack on the substrate carbonyl carbon occurs simultaneously with proton transfer from water to the P-site A76 O2'. This single proton transfer is also consistent with the measured KSIE of 4.1 and linear proton inventory plot. ${ }^{9}$ The proposed mechanism further supports the role of the Gln 240 in stabilizing the partially negative substrate carbonyl oxygen and in orienting the nucleophile for efficient attack on the ester carbon atom. ${ }^{1}$ Overall, this substrate-assisted base catalysis pathway appears to be consistent with all available experimental data. The question then naturally arises why such a mechanism does not seem to be operational in peptide bond formation, where the reaction does not show the same linear $\mathrm{pH}$ dependence as in peptide release. ${ }^{7,35}$ The reason for this could be that one proton has already been removed from the system, when the attacking Asite amine becomes deprotonated, and no more protons can be lost if an ionized leaving group is to be prevented. In the hydrolysis reaction, on the other hand, the O3' leaving group can still be protonated by the carboxylic acid moiety of the leaving peptide, which is a downhill process.

\section{ASSOCIATED CONTENT}

\section{Supporting Information}

The Supporting Information is available free of charge on the ACS Publications website at DOI: 10.1021/acscatal.6b02842.

Comparison of the orientation of the substrate in crystal structures with different optimized transition states and the coordinates of all optimized states (PDF) 


\section{AUTHOR INFORMATION}

\section{Corresponding Authors}

*E-mail for F.H.: fahmi.himo@su.se.

*E-mail for J.Å: aqvist@xray.bmc.uu.se.

\section{ORCID}

Johan Åqvist: 0000-0003-2091-0610

Notes

The authors declare no competing financial interest.

\section{ACKNOWLEDGMENTS}

Support from the Swedish Research Council (VR), the Knut and Alice Wallenberg Foundation, the eSSENCE e-science initiative, and the Swedish National Infrastructure for Computing (SNIC) is gratefully acknowledged.

\section{ABBREVIATIONS}

DFT, density functional theory; RF, release factor; PTC, peptidyl transferase center; KSIE, kinetic solvent isotope effect; TS, transition state

\section{REFERENCES}

(1) Trobro, S.; Åqvist, J. Mol. Cell 2007, 27, 758-766.

(2) Laurberg, M.; Asahara, H.; Korostelev, A.; Zhu, J.; Trakhanov, S.; Noller, H. F. Nature 2008, 454, 852-857.

(3) Korostelev, A.; Asahara, H.; Lancaster, L.; Laurberg, M.; Hirschi, A.; Zhu, J.; Trakhanov, S.; Scott, W. G.; Noller, H. F. Proc. Natl. Acad. Sci. U. S. A. 2008, 105, 19684-19689.

(4) Weixlbaumer, A.; Jin, H.; Neubauer, C.; Voorhees, R. M.; Petry, S.; Kelley, A. C.; Ramakrishnan, V. Science 2008, 322, 953-956.

(5) Trobro, S.; Åqvist, J. Biochemistry 2009, 48, 11296-11303.

(6) Wallin, G.; Åqvist, J. Proc. Natl. Acad. Sci. U. S. A. 2010, 107, 1888-1893.

(7) Johansson, M.; Ieong, K.-W.; Trobro, S.; Strazewski, P.; Åqvist, J.; Pavlov, M. Y.; Ehrenberg, M. Proc. Natl. Acad. Sci. U. S. A. 2011, 108, $79-84$.

(8) Åqvist, J.; Lind, C.; Sund, J.; Wallin, G. Curr. Opin. Struct. Biol. 2012, 22, 815-823.

(9) Kuhlenkoetter, S.; Wintermeyer, W.; Rodnina, M. V. Nature 2011, 476, 351-354.

(10) Shaw, J. J.; Trobro, S.; He, S. L.; Åqvist, J.; Green, R. Chem. Biol. 2012, 19, 983-993.

(11) Indrisiunaite, G.; Pavlov, M. Y.; Heurgué-Hamard, V.; Ehrenberg, M. J. Mol. Biol. 2015, 427, 1848-1860.

(12) Åström, H.; Limén, E.; Strömberg, R. J. Am. Chem. Soc. 2004, 126, 14710-14711.

(13) Acosta-Silva, C.; Bertran, J.; Branchadell, V.; Oliva, A. J. Phys. Chem. B 2013, 117, 3503-3515.

(14) Acosta-Silva, C.; Bertran, J.; Branchadell, V.; Oliva, A. J. J. Phys. Chem. B 2014, 118, 5717-5729.

(15) Monajemi, H.; Zain, S. M.; Wan Abdullah, W. A. T. Comput. Theor. Chem. 2011, 976, 148-152.

(16) Wang, Q.; Gao, J.; Liu, Y.; Liu, C. Chem. Phys. Lett. 2010, 501, $113-117$.

(17) Polikanov, Y. S.; Steitz, T. A.; Innis, C. A. Nat. Struct. Mol. Biol. 2014, 21, 787-793.

(18) Schmeing, T. M.; Huang, K. S.; Strobel, S. A.; Steitz, T. A. Nature 2005, 438, 520-524.

(19) Schmeing, T. M.; Huang, K. S.; Kitchen, D. E.; Strobel, S. A.; Steitz, T. A. Mol. Cell 2005, 20, 437-448.

(20) Jin, H.; Kelley, A. C.; Loakes, D.; Ramakrishnan, V. Proc. Natl. Acad. Sci. U. S. A. 2010, 107, 8593-8598.

(21) Frisch, M. J.; Trucks, G. W.; Schlegel, H. B.; Scuseria, G. E.; Robb, M. A.; Cheeseman, J. R.; Scalmani, G.; Barone, V.; Mennucci, B.; Petersson, G. A.; Nakatsuji, H.; Caricato, M.; Li, X.; Hratchian, H. P.; Izmaylov, A. F.; Bloino, J.; Zheng, G.; Sonnenberg, J. L.; Hada, M.; Ehara, M.; Toyota, K.; Fukuda, R.; Hasegawa, J.; Ishida, M.; Nakajima,
T.; Honda, Y.; Kitao, O.; Nakai, H.; Vreven, T.; Montgomery, J. A., Jr.; Peralta, J. E.; Ogliaro, F.; Bearpark, M.; Heyd, J. J.; Brothers, E.; Kudin, K. N.; Staroverov, V. N.; Kobayashi, R.; Normand, J.; Raghavachari, K.; Rendell, A.; Burant, J. C.; Iyengar, S. S.; Tomasi, J.; Cossi, M.; Rega, N.; Millam, J. M.; Klene, M.; Knox, J. E.; Cross, J. B.; Bakken, V.; Adamo, C.; Jaramillo, J.; Gomperts, R.; Stratmann, R. E.; Yazyev, O.; Austin, A. J.; Cammi, R.; Pomelli, C.; Ochterski, J. W.; Martin, R. L.; Morokuma, K.; Zakrzewski, V. G.; Voth, G. A.; Salvador, P.; Dannenberg, J. J.; Dapprich, S.; Daniels, A. D.; Farkas, Ö.; Foresman, J. B.; Ortiz, J. V; Cioslowski, J.; Fox, D. J. Gaussian 09, Revision D.01; Gaussian Inc., Wallingford, CT, 2009.

(22) Zhao, Y.; Truhlar, D. G. Theor. Chem. Acc. 2008, 120, 215-241.

(23) Marenich, A. V.; Cramer, C. J.; Truhlar, D. G. J. Phys. Chem. B 2009, 113, 6378-6396.

(24) Youngman, E. M.; Brunelle, J. L.; Kochaniak, A. B.; Green, R. Cell 2004, 117, 589-599.

(25) Frolova, L. Y.; Tsivkovskii, R. Y.; Sivolobova, G. F.; Oparina, N. Y.; Serpinsky, O. I.; Blinov, V. M.; Tatkov, S. I.; Kisselev, L. L. RNA 1999, 5, 1014-1020.

(26) Zavialov, A. V.; Mora, L.; Buckingham, R. H.; Ehrenberg, M. Mol. Cell 2002, 10, 789-798.

(27) Shaw, J. J.; Green, R. Mol. Cell 2007, 28, 458-467.

(28) Trobro, S.; Åqvist, J. Biochemistry 2006, 45, 7049-7056.

(29) Swiderek, K.; Marti, S.; Tuñón, I.; Moliner, V.; Bertran, J. J. Am. Chem. Soc. 2015, 137, 12024-12034.

(30) Trobro, S.; Åqvist, J. Proc. Natl. Acad. Sci. U. S. A. 2005, 102, 12395-12400.

(31) Sharma, P. K.; Xiang, Y.; Kato, M.; Warshel, A. Biochemistry 2005, 44, 11307-11314.

(32) Wolfenden, R. Biochemistry 1963, 2, 1090-1092.

(33) Mora-Diez, N.; Egorova, Y.; Plommer, H.; Tremaine, P. R. RSC Adv. 2015, 5, 9097-9109.

(34) Ballinger, P.; Long, F. A. J. Am. Chem. Soc. 1960, 82, 795-798.

(35) Bieling, P.; Beringer, M.; Adio, S.; Rodnina, M. V. Nat. Struct. Mol. Biol. 2006, 13, 423-428. 